Homology, Homotopy and Applications, vol.15(2), 2013, pp.185-194

\title{
CHEVALLEY-EILENBERG HOMOLOGY OF CROSSED MODULES OF LIE ALGEBRAS IN LOWER DIMENSIONS
}

\author{
GURAM DONADZE AND MANUEL LADRA
}

(communicated by Graham Ellis)

\begin{abstract}
In this paper we prove a five term exact sequence connecting in lower dimensions the Chevalley-Eilenberg homologies of the crossed module of Lie algebras $(\mathfrak{m}, \mathfrak{g}, \mu)$ and of the Lie algebra $\mathfrak{g} / \operatorname{Im}(\mu)$. Moreover, a relationship between the ChevalleyEilenberg homology with coefficients and the homology of a crossed module of Lie algebras is established.
\end{abstract}

\section{Introduction}

Crossed modules of Lie algebras, which are simultaneous generalisations of ideals and modules over Lie algebras, were introduced by Kassel and Loday (see [11]), in order to give an interpretation of the third relative Chevalley-Eilenberg cohomology of Lie algebras. Crossed modules of Lie algebras are algebraic objects equivalent to simplicial Lie algebras with the associated Moore complex of length 1 (see, e.g., $[\mathbf{8}, \mathbf{9}]$ ). A first approach to an internal (low dimensional) homology theory of crossed modules of Lie algebras was done in [5]. In [4], the authors studied the homology theory of crossed modules of Lie algebras in the spirit of $[\mathbf{3}, \mathbf{1 0}]$. In particular, the authors checked that the category of crossed modules of Lie algebras is tripleable and proved that the natural homology theory obtained from this triple (called cotriple homology) can be determined by small complexes formed from the standard Chevalley-Eilenberg complex of Lie algebras. In [7] it is shown that lower dimensional cyclic homology groups of associative algebras can be described in terms of the cotriple homology of crossed modules of Lie algebras.

The present paper provides answers to some questions posed in [4]. In particular, the existence of a five term exact sequence connecting the low-dimensional ChevalleyEilenberg homologies of crossed modules and their cokernel Lie algebras is proved. The analogous result for the cyclic and Hochschild homologies of crossed modules of associative algebras is given in [6], and its proof is based on using of the EilenbergZilber theorem and the Künneth formula, which are not valid in the case mentioned above. Moreover, a relationship between the homology of a crossed module of Lie algebras and the Chevalley-Eilenberg homology of Lie algebras with coefficients is established in the paper.

Received March 14, 2013, revised March 12, 2013; published on November 27, 2013. 2010 Mathematics Subject Classification: 17B40, 17B56, 18G50, 18G60, 19 D55.

Key words and phrases: crossed modules of Lie algebras, Chevalley-Eilenberg homology. Article available at http://intlpress.com/HHA/v15/n2/a12 and doi:10.4310/HHA.2013.v15.n2.a12 Copyright (C) 2013, International Press. Permission to copy for private use granted. 


\section{Notations and Conventions}

Throughout the paper we fix a field $\mathbf{k}$. All tensor and exterior products are over $\mathbf{k}$. All Lie algebras and vector spaces we deal with are also over $\mathbf{k}$. Lie bracket is denoted by [, ]. Given a Lie algebra $\mathfrak{g}$ and a right module $V$ over $\mathfrak{g}$, denote by $[V, \mathfrak{g}]$ the vector subspace of $V$ generated by the elements $[v, g]$ for all $v \in V$ and $g \in \mathfrak{g}$, where $[-,-]: V \times \mathfrak{g} \rightarrow V$ is the action.

\section{Acknowledgments}

The authors were partially supported by the Ministerio de Ciencia e Innovación (Spain), grant MTM2009-14464-C02 (European FEDER support included).

\section{Chevalley-Eilenberg homology of crossed modules of Lie algebras}

A crossed module of Lie algebras $(\mathfrak{m}, \mathfrak{g}, \mu)$ consists of a Lie homomorphism $\mu: \mathfrak{m}$ $\rightarrow \mathfrak{g}$ together with a Lie action of $\mathfrak{g}$ on $\mathfrak{m}$ which is a k-linear map $\mathfrak{g} \times \mathfrak{m} \rightarrow \mathfrak{m},(g, m) \mapsto$ ${ }^{g} m$, satisfying

$$
{ }^{\left[g, g^{\prime}\right]} m={ }^{g}\left(g^{\prime} m\right)-{ }^{g^{\prime}}\left({ }^{g} m\right), \quad{ }^{g}\left[m, m^{\prime}\right]=\left[{ }^{g} m, m^{\prime}\right]+\left[m,{ }^{g} m^{\prime}\right],
$$

such that the following conditions hold:

$$
\begin{aligned}
\mu\left({ }^{g} m\right) & =[g, \mu(m)], \\
\mu(m) & m^{\prime}=\left[m, m^{\prime}\right] \quad \text { (Peiffer identity), }
\end{aligned}
$$

for all $m, m^{\prime} \in \mathfrak{m}$ and $g \in \mathfrak{g}$. One easily sees that $\operatorname{Ker}(\mu)$ is contained in the center of $\mathfrak{m}$. Moreover, the image of $\mu, \operatorname{Im}(\mu)$, is necessarily an ideal in $\mathfrak{g}$ and $\operatorname{Ker}(\mu)$ is a module over $\operatorname{Coker}(\mu)=\mathfrak{g} / \operatorname{Im}(\mu)$.

A common example of crossed module of Lie algebras is an inclusion homomorphism $\mathfrak{n} \hookrightarrow \mathfrak{g}$ for any Lie algebra $\mathfrak{g}$ and its ideal $\mathfrak{n}$. Another common instance is the trivial homomorphism $0: V \rightarrow \mathfrak{g}, v \mapsto 0$, for any $\mathfrak{g}$-module $V$, where $V$ is considered as Lie algebra with trivial Lie bracket. For other examples of Lie algebra crossed modules the reader can see $[\mathbf{1}, \mathbf{2}, \mathbf{1 2}]$.

A morphism of crossed modules of Lie algebras $(\alpha, \beta):(\mathfrak{m}, \mathfrak{g}, \mu) \rightarrow\left(\mathfrak{m}^{\prime}, \mathfrak{g}^{\prime}, \mu^{\prime}\right)$ consists of Lie homomorphisms $\alpha: \mathfrak{m} \rightarrow \mathfrak{m}^{\prime}, \beta: \mathfrak{g} \rightarrow \mathfrak{g}^{\prime}$ such that $\mu^{\prime} \alpha(m)=\beta \mu(m)$ and $\alpha\left({ }^{g} m\right)={ }^{\beta(g)} \alpha(m)$ for all $m \in \mathfrak{m}$ and $g \in \mathfrak{g}$. In this way we get the category of crossed modules of Lie algebras.

Given any crossed module of Lie algebras $(\mathfrak{m}, \mathfrak{g}, \mu)$ we can form the semidirect product of Lie algebras, $\mathfrak{m} \rtimes \mathfrak{g}$, with the underlying vector space $\mathfrak{m} \oplus \mathfrak{g}$ endowed with the Lie algebra bracket defined by the formula

$$
\left[(m, g),\left(m^{\prime}, g^{\prime}\right)\right]=\left(\left[m, m^{\prime}\right]+{ }^{g} m^{\prime}-{ }^{g^{\prime}} m,\left[g, g^{\prime}\right]\right),
$$

for all $(m, g),\left(m^{\prime}, g^{\prime}\right) \in \mathfrak{m} \rtimes \mathfrak{g}$. Moreover, there are Lie homomorphisms $s: \mathfrak{m} \rtimes \mathfrak{g} \rightarrow \mathfrak{g}$, $(m, g) \mapsto g$ and $t: \mathfrak{m} \rtimes \mathfrak{g} \rightarrow \mathfrak{g}, \quad(m, g) \mapsto \mu(m)+g$, and a binary operation $\left(m^{\prime}, g^{\prime}\right) \circ(m, g)=\left(m+m^{\prime}, g\right)$ defined for any pair $(m, g),\left(m^{\prime}, g^{\prime}\right) \in \mathfrak{m} \rtimes \mathfrak{g}$ such that $\mu(m)+g=g^{\prime}$. This composition $\circ$ with the source map $s$ and target map $t$ constitutes an internal category in the category of Lie algebras. The nerve of its category structure 
forms the simplicial Lie algebra $\mathcal{N}_{*}(\mathfrak{m}, \mathfrak{g}, \mu)$, where $\mathcal{N}_{*}(\mathfrak{m}, \mathfrak{g}, \mu)=\mathfrak{m} \rtimes(\cdots(\mathfrak{m} \rtimes \mathfrak{g}) \cdots)$ with $n$ semidirect factors of $\mathfrak{m}$, and face and degeneracy homomorphisms are defined by

$$
\begin{aligned}
d_{0}\left(m_{1}, \ldots, m_{n}, g\right) & =\left(m_{2}, \ldots, m_{n}, g\right), \\
d_{i}\left(m_{1}, \ldots, m_{n}, g\right) & =\left(m_{1}, \ldots, m_{i}+m_{i+1}, \ldots, m_{n}, g\right), \quad 0<i<n, \\
d_{n}\left(m_{1}, \ldots, m_{n}, g\right) & =\left(m_{1}, \ldots, m_{n-1}, \mu\left(m_{n}\right)+g\right), \\
s_{i}\left(m_{1}, \ldots, m_{n}, g\right) & =\left(m_{1}, \ldots, m_{i}, 0, m_{i+1}, \ldots, m_{n}, g\right), \quad 0 \leqslant i \leqslant n .
\end{aligned}
$$

This simplicial Lie algebra is called the nerve of the crossed module of Lie algebras and its Moore complex is trivial in dimensions $\geqslant 2$. In fact its Moore complex is just the original crossed module up to isomorphism with $\mathfrak{m}$ in dimension 1 and $\mathfrak{g}$ in dimension 0 .

\section{Chevalley-Eilenberg homology}

Given a Lie algebra $\mathfrak{g}$ and a (right) $\mathfrak{g}$-module $V$, the standard Chevalley-Eilenberg complex, $C^{C E}(\mathfrak{g}, V)$, has the following form:

$$
\cdots \rightarrow V \otimes \wedge^{n} \mathfrak{g} \stackrel{\partial_{n}}{\longrightarrow} V \otimes \wedge^{n-1} \mathfrak{g} \stackrel{\partial_{n-1}}{\longrightarrow} \cdots \stackrel{\partial_{3}}{\longrightarrow} V \otimes \wedge^{2} \mathfrak{g} \stackrel{\partial_{2}}{\longrightarrow} V \otimes \mathfrak{g} \stackrel{\partial_{1}}{\longrightarrow} V,
$$

where the boundary map $\partial_{n}$ is given by the formula

$$
\begin{aligned}
\partial_{n}\left(v \otimes g_{1} \wedge \cdots \wedge g_{n}\right) & =\sum_{i=1}^{n}(-1)^{i+1}\left[v, g_{i}\right] \otimes g_{1} \wedge \cdots \wedge \widehat{g}_{i} \wedge \cdots \wedge g_{n} \\
& +\sum_{1 \leqslant i<j \leqslant n}(-1)^{i+j} v \otimes\left[g_{i}, g_{j}\right] \wedge g_{1} \wedge \cdots \wedge \widehat{g}_{i} \wedge \cdots \wedge \widehat{g_{j}} \wedge \cdots \wedge g_{n} .
\end{aligned}
$$

The Chevalley-Eilenberg homology of the Lie algebra $\mathfrak{g}$ with coefficients in $V, H_{*}(\mathfrak{g}, V)$ is defined to be the homology of the complex $C^{C E}(\mathfrak{g}, V)$. If $V=\mathbf{k}$ is considered as a trivial $\mathfrak{g}$-module, then the Chevalley-Eilenberg complex is denoted by $C^{C E}(\mathfrak{g})$ and its homology by $H_{*}(\mathfrak{g})$.

Given a simplicial Lie algebra $\mathfrak{g}_{*}$, the Chevalley-Eilenberg homology extends to $\mathfrak{g}_{*}$ in a natural way (see, e.g., [4]). Namely, applying the Chevalley-Eilenberg complex, $C^{C E}(-)$, dimensional-wise to the simplicial Lie algebra $\mathfrak{g}_{*}$, we arrive at the following bicomplex

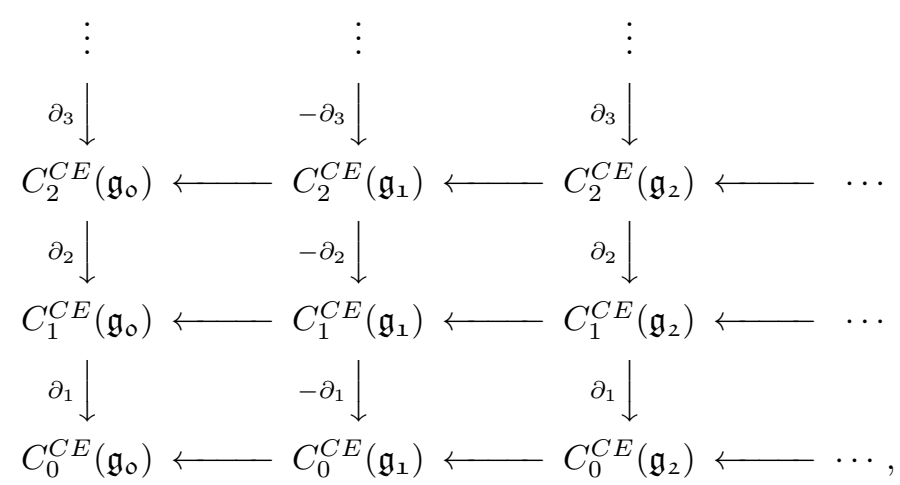


denoted by $C^{C E}\left(\mathfrak{g}_{*}\right)$, where the horizontal differentials are obtained by taking alternating sums. The Chevalley-Eilenberg homology of the simplicial Lie algebra $\mathfrak{g}_{*}$ is defined by the formula

$$
H_{n}\left(\mathfrak{g}_{*}\right)=H_{n}\left(\operatorname{Tot}\left(C^{C E}\left(\mathfrak{g}_{*}\right)\right)\right), n \geqslant 0 .
$$

Given a crossed module of Lie algebras $(\mathfrak{m}, \mathfrak{g}, \mu)$, denote by $C^{C E}(\mathfrak{m}, \mathfrak{g}, \mu)$ the total complex $\operatorname{Tot}\left(C^{C E}\left(\mathcal{N}_{*}(\mathfrak{m}, \mathfrak{g}, \mu)\right)\right)$. Then the Chevalley-Eilenberg homology of $(\mathfrak{m}, \mathfrak{g}, \mu)$ is defined by the formula

$$
H_{n}(\mathfrak{m}, \mathfrak{g}, \mu)=H_{n}\left(C^{C E}(\mathfrak{m}, \mathfrak{g}, \mu)\right), \quad n \geqslant 0 .
$$

In other words, $H_{n}(\mathfrak{m}, \mathfrak{g}, \mu)$ is defined as the Chevalley-Eilenberg homology of the nerve $\mathcal{N}_{*}(\mathfrak{m}, \mathfrak{g}, \mu)$.

\section{Five term exact sequence}

The aim of this section is to prove the following:

Proposition 3.1. Let $(\mathfrak{m}, \mathfrak{g}, \mu)$ be a crossed module of Lie algebras. Then

$$
H_{0}(\mathfrak{m}, \mathfrak{g}, \mu)=\mathbf{k} \quad \text { and } \quad H_{1}(\mathfrak{m}, \mathfrak{g}, \mu)=\operatorname{Coker}(\mu) /[\operatorname{Coker}(\mu), \operatorname{Coker}(\mu)] .
$$

Moreover, if characteristic of $\mathbf{k}$ is not 2 (i.e., $1 / 2 \in \mathbf{k}$ ), then there is an exact sequence of vector spaces

$$
\begin{aligned}
H_{3}(\mathfrak{m}, \mathfrak{g}, \mu) \rightarrow H_{3}(\operatorname{Coker}(\mu)) \rightarrow \operatorname{Ker}(\mu) /[\operatorname{Ker}(\mu), \mathfrak{g}] \rightarrow H_{2}(\mathfrak{m}, \mathfrak{g}, \mu) \rightarrow & H_{2}(\operatorname{Coker}(\mu)) \rightarrow 0 .
\end{aligned}
$$

First, a few auxiliary lemmas will be proved. Define the simplicial vector spaces $X_{*}, \widehat{X}_{*}$ and $\bar{X}_{*}$ in the following way:

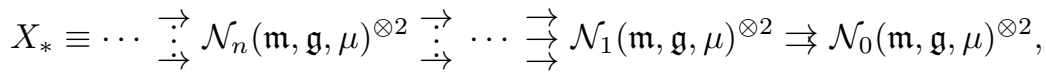

$$
\begin{aligned}
& \widehat{X}_{*} \equiv \cdots \underset{\dot{m}}{\overrightarrow{!}} \mathcal{N}_{n}(\mathfrak{m}, \mathfrak{g}, \mu)^{\wedge^{2}} \stackrel{\vec{\vdots}}{\rightarrow} \cdots \underset{\rightarrow}{\rightarrow} \mathcal{N}_{1}(\mathfrak{m}, \mathfrak{g}, \mu)^{\wedge^{2}} \rightrightarrows \mathcal{N}_{0}(\mathfrak{m}, \mathfrak{g}, \mu)^{\wedge^{2}} \\
& \bar{X}_{*} \equiv \operatorname{Ker}\left\{X_{*} \rightarrow \widehat{X}_{*}\right\},
\end{aligned}
$$

where face and degeneracy homomorphisms are defined componentwise.

Lemma 3.2. If $1 / 2 \in \mathbf{k}$, then there is a short exact sequence of homotopy groups

$$
0 \rightarrow \pi_{0}\left(\bar{X}_{*}\right) \rightarrow \pi_{0}\left(X_{*}\right) \rightarrow \pi_{0}\left(\widehat{X}_{*}\right) \rightarrow 0 .
$$

Proof. We have the following short exact sequence of simplicial vector spaces:

$$
0 \rightarrow \bar{X}_{*} \rightarrow X_{*} \rightarrow \widehat{X}_{*} \rightarrow 0
$$

The corresponding long exact sequence of homotopy groups

$$
\cdots \rightarrow \pi_{1}\left(\bar{X}_{*}\right) \rightarrow \pi_{1}\left(X_{*}\right) \rightarrow \pi_{1}\left(\widehat{X}_{*}\right) \rightarrow \pi_{0}\left(\bar{X}_{*}\right) \rightarrow \pi_{0}\left(X_{*}\right) \rightarrow \pi_{0}\left(\widehat{X}_{*}\right) \rightarrow 0
$$

implies that it suffices to show the injectivity of the homomorphism $\pi_{0}\left(\bar{X}_{*}\right) \rightarrow \pi_{0}\left(X_{*}\right)$ which we denote by $i$. To finish the proof we construct a homomorphism $\tau: \pi_{0}\left(X_{*}\right) \rightarrow$ $\pi_{0}\left(\bar{X}_{*}\right)$ such that $\tau i=1_{\pi_{0}\left(\bar{X}_{*}\right)}$. 
Direct calculus gives

$$
\begin{aligned}
& \pi_{0}\left(X_{*}\right)=(\mathfrak{g} \otimes \mathfrak{g}) /(\operatorname{Im}(\mu) \otimes \mathfrak{g}+\mathfrak{g} \otimes \operatorname{Im}(\mu)), \\
& \pi_{0}\left(\bar{X}_{*}\right)=\frac{\{\text { the submodule of } \mathfrak{g} \otimes \mathfrak{g} \text { generated by } g \otimes g, g \in \mathfrak{g}\}}{\{\text { the submodule of } \mathfrak{g} \otimes \mathfrak{g} \text { generated by } g \otimes x+x \otimes g, g \in \mathfrak{g}, x \in \operatorname{Im}(\mu)\}} .
\end{aligned}
$$

Take $g_{1} \otimes g_{2} \in \mathfrak{g} \otimes \mathfrak{g}$ and assume that

$$
g_{1} \otimes g_{2} \stackrel{\tau}{\longmapsto} \frac{1}{2}\left(g_{1} \otimes g_{2}+g_{2} \otimes g_{1}\right) .
$$

We easily checks that $\tau$ is well defined on $\pi_{0}\left(X_{*}\right)$ and $\tau i=1_{\pi_{0}\left(\bar{X}_{*}\right)}$.

It is well known that each simplicial vector space gives rise to a chain complex whose objects are the same and the differentials are obtained by alternating sum of the face homomorphisms. Denote by $\left(X_{*}, \partial_{*}\right),\left(\widehat{X}_{*}, \widehat{\partial}_{*}\right)$ and $\left(\bar{X}_{*}, \bar{\partial}_{*}\right)$ the corresponding chain complexes of the simplicial vector spaces $X_{*}, \widehat{X}_{*}$ and $\bar{X}_{*}$, respectively.

Lemma 3.3. If $1 / 2 \in \mathbf{k}$, then

$$
\begin{aligned}
& H_{1}\left(\widehat{X}_{*}, \widehat{\partial}_{*}\right) \\
& =\frac{\left\{\text { the submodule of } \wedge^{2}(\mathfrak{m} \rtimes \mathfrak{g}) \text { generated by } x \wedge y, x \in \operatorname{Ker}(\mu), y \in \mathfrak{m} \rtimes \mathfrak{g}\right\}+\operatorname{Im} \widehat{\partial}_{2}}{\operatorname{Im} \widehat{\partial}_{2}} .
\end{aligned}
$$

Proof. We have the short exact sequence of complexes

$$
0 \rightarrow\left(\bar{X}_{*}, \bar{\partial}_{*}\right) \rightarrow\left(X_{*}, \partial_{*}\right) \rightarrow\left(\widehat{X}_{*}, \widehat{\partial}_{*}\right) \rightarrow 0,
$$

which gives rise to the long homology exact sequence

$$
\cdots \rightarrow H_{1}\left(X_{*}, \partial_{*}\right) \rightarrow H_{1}\left(\widehat{X}_{*}, \widehat{\partial}_{*}\right) \rightarrow H_{0}\left(\bar{X}_{*}, \bar{\partial}_{*}\right) \rightarrow H_{0}\left(X_{*}, \partial_{*}\right) \rightarrow H_{0}\left(\widehat{X}_{*}, \widehat{\partial}_{*}\right) \rightarrow 0 .
$$

Since the homotopy groups of simplicial vector space are isomorphic to the homology groups of the corresponding chain complex, by the previous lemma $H_{1}\left(X_{*}, \partial_{*}\right) \rightarrow$ $H_{1}\left(\widehat{X}_{*}, \widehat{\partial}_{*}\right)$ is an epimorphism. Thus, to finish the proof it suffices to show that the following natural homomorphism

$$
\operatorname{Ker}(\mu) \otimes(\mathfrak{m} \rtimes \mathfrak{g})+(\mathfrak{m} \rtimes \mathfrak{g}) \otimes \operatorname{Ker}(\mu) \rightarrow H_{1}\left(X_{*}, \partial_{*}\right)
$$

is an epimorphism. The latter follows from the Eilenberg-Zilber theorem and the Künneth formula.

Proof of Proposition 3.1. Consider the bicomplex $C^{C E}\left(\mathcal{N}_{*}(\mathfrak{m}, \mathfrak{g}, \mu)\right)$ in Figure 1. There is a spectral sequence of the first quadrant

$$
E_{p q}^{1}=H_{q}\left(C_{p}^{C E}\left(\mathcal{N}_{*}(\mathfrak{m}, \mathfrak{g}, \mu)\right)\right) \Rightarrow H_{p+q}(\mathfrak{m}, \mathfrak{g}, \mu) .
$$

We have

$$
\begin{aligned}
& E_{p 0}^{1}=C_{p}^{C E}(\operatorname{Coker}(\mu)) \quad \text { for all } p \geqslant 0, \\
& E_{0 q}^{1}=0 \quad \text { when } q \geqslant 1, \\
& E_{1 q}^{1}=0 \quad \text { when } q \geqslant 2 \text { and } \quad E_{11}^{1}=\operatorname{Ker}(\mu) .
\end{aligned}
$$




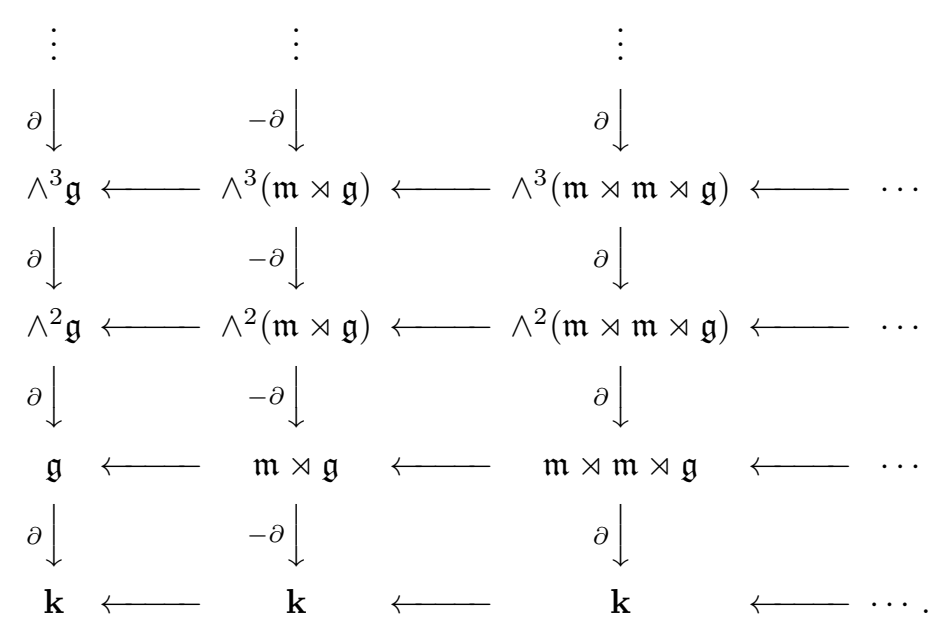

Figure 1: The bicomplex $C^{C E}\left(\mathcal{N}_{*}(\mathfrak{m}, \mathfrak{g}, \mu)\right)$.

Hence

$$
\begin{aligned}
& E_{p 0}^{\infty}=H_{p}(\operatorname{Coker}(\mu)) \text { when } p \leqslant 2, \quad \text { and } E_{p 0}^{2}=H_{p}(\operatorname{Coker}(\mu)) \text { when } p \geqslant 3, \\
& E_{0 q}^{\infty}=0 \text { when } q \geqslant 1, \text { and } E_{1 q}^{\infty}=0 \text { when } q \geqslant 2 .
\end{aligned}
$$

This implies

$$
\begin{aligned}
& H_{0}(\mathfrak{m}, \mathfrak{g}, \mu)=H_{0}(\operatorname{Coker}(\mu))=\mathbf{k}, \\
& H_{1}(\mathfrak{m}, \mathfrak{g}, \mu)=H_{1}(\operatorname{Coker}(\mu))=\operatorname{Coker}(\mu) /[\operatorname{Coker}(\mu), \operatorname{Coker}(\mu)] .
\end{aligned}
$$

Thus, the first part of the proposition is proved.

Now we calculate $E_{11}^{2}=\operatorname{Coker}\left\{E_{21}^{1} \rightarrow E_{11}^{1}\right\}$. By the definition, $E_{21}^{1}$ is exactly $H_{1}\left(\widehat{X}_{*}, \widehat{\partial}_{*}\right)$. Therefore, by the previous lemma we will have

$$
\begin{aligned}
E_{11}^{2} & =\operatorname{Coker}\left\{E_{21}^{1} \rightarrow E_{11}^{1}\right\}=\operatorname{Coker}\left\{H_{1}\left(\widehat{X}_{*}, \widehat{\partial}_{*}\right) \rightarrow \operatorname{Ker}(\mu)\right\} \\
& =\operatorname{Ker}(\mu) /[\operatorname{Ker}(\mu), \mathfrak{m} \rtimes \mathfrak{g}]=\operatorname{Ker}(\mu) /[\operatorname{Ker}(\mu), \mathfrak{g}] .
\end{aligned}
$$

Since $E_{02}^{1}=0$, we have the following exact sequence:

$$
0 \rightarrow E_{30}^{\infty} \rightarrow E_{30}^{2} \rightarrow E_{11}^{2} \rightarrow E_{11}^{\infty} \rightarrow 0
$$

Hence, according to the formulas mentioned above, we get an exact sequence

$$
0 \rightarrow E_{30}^{\infty} \rightarrow H_{3}(\operatorname{Coker}(\mu)) \rightarrow \operatorname{Ker}(\mu) /[\operatorname{Ker}(\mu), \mathfrak{g}] \rightarrow E_{11}^{\infty} \rightarrow 0 .
$$

Moreover, we have an epimorphism

$$
H_{3}(\mathfrak{m}, \mathfrak{g}, \mu) \rightarrow E_{30}^{\infty}
$$

and an exact sequence

$$
0 \rightarrow E_{11}^{\infty} \rightarrow H_{2}(\mathfrak{m}, \mathfrak{g}, \mu) \rightarrow E_{20}^{\infty} \rightarrow 0
$$

Since $E_{20}^{\infty}=H_{2}(\operatorname{Coker}(\mu)),(1)(2)$ and (3) imply the required result.

Corollary 3.4. Let $\mathbf{k}$ be a field, $1 / 2 \in \mathbf{k}$ and $V$ be $a \mathbf{k}$-module with trivial Lie bracket. Then $\mathrm{H}_{2}(V, 0,0)=V$. 
We will later show the last isomorphism without the restriction $1 / 2 \in \mathbf{k}$.

\section{Relationship to the Chevalley-Eilenberg homology with coefficients}

Given a Lie algebra $\mathfrak{g}$ and a $\mathfrak{g}$-module $V$, there is a crossed module defined by the trivial homomorphism $0: V \rightarrow \mathfrak{g}$, which we denote by $(V, \mathfrak{g}, 0)$. We have a natural morphism of crossed modules of Lie algebras $\left(0,1_{\mathfrak{g}}\right):(0, \mathfrak{g}, 0) \rightarrow(V, \mathfrak{g}, 0)$, which induces a homomorphism of chain complexes

$$
\left(0,1_{\mathfrak{g}}\right)_{*}: C^{C E}(0, \mathfrak{g}, 0) \rightarrow C^{C E}(V, \mathfrak{g}, 0) .
$$

This homomorphism is injective, since the ground ring $\mathbf{k}$ is a field. Define the chain complex $\Theta(V, \mathfrak{g})$ from the following short exact sequence of complexes:

$$
0 \rightarrow C^{C E}(0, \mathfrak{g}, 0) \stackrel{\left(0,1_{g}\right)_{*}}{\longrightarrow} C^{C E}(V, \mathfrak{g}, 0) \rightarrow \Theta(V, \mathfrak{g}) \rightarrow 0 .
$$

It is easy to calculate that

$$
\begin{aligned}
& H_{0}(\Theta(V, \mathfrak{g}))=H_{1}(\Theta(V, \mathfrak{g}))=0 \quad \text { and } \\
& \left.H_{2}(\Theta(V, \mathfrak{g}))=V /[V, \mathfrak{g}]=H_{0}(\mathfrak{g}, V) \quad \text { (see [4] }\right) .
\end{aligned}
$$

Proposition 4.1. For any integer $n \geqslant 0$ there is a homomorphism

$$
H_{n+2}(\Theta(V, \mathfrak{g})) \rightarrow H_{n}(\mathfrak{g}, V) .
$$

Moreover, this homomorphism is an isomorphism for $n=0,1$ and an epimorphism for $n=2$.

Proof. Define a bicomplex $X_{* *}=C^{C E}\left(\mathcal{N}_{*}(V, \mathfrak{g}, 0)\right) / C^{C E}\left(\mathcal{N}_{*}(0, \mathfrak{g}, 0)\right)$. By definition,

$$
H_{n}(\Theta(V, \mathfrak{g}))=H_{n}\left(\operatorname{Tot}\left(X_{* *}\right)\right), \quad n \geqslant 0
$$

There is a spectral sequence of the first quadrant

$$
E_{p q}^{1} \Rightarrow H_{q}\left(X_{* p}\right) .
$$

The bicomplex $C^{C E}\left(\mathcal{N}_{*}(V, \mathfrak{g}, 0)\right)$ has the following form:

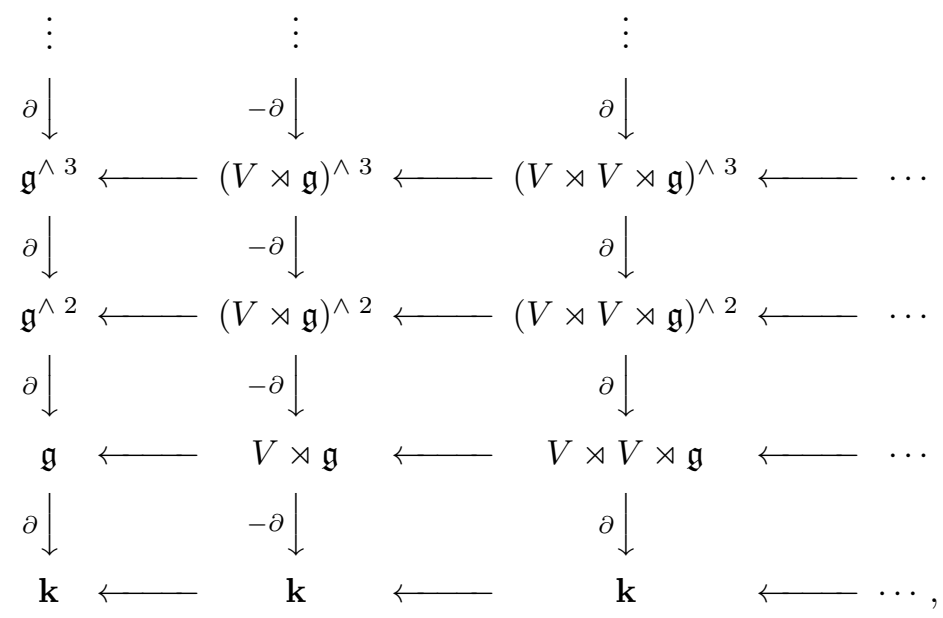


while $C^{C E}\left(\mathcal{N}_{*}(0, \mathfrak{g}, 0)\right)$ has the following form:

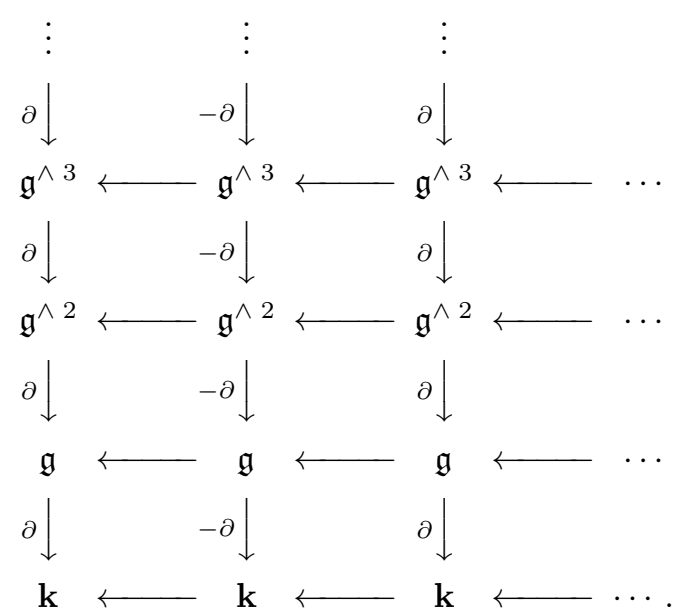

Consequently, $X_{* 0}=X_{0 *}=0$. This implies $E_{p 0}^{1}=0$ for all $p \geqslant 0$. Therefore, for all $n \geqslant 0$, there exists a homomorphism

$$
H_{n+1}\left(\operatorname{Tot}\left(X_{* *}\right)\right) \rightarrow E_{n 1}^{2} .
$$

Moreover, it is routine to check that $E_{n 1}^{1}=V \otimes \mathfrak{g}^{\wedge} n-1, n \geqslant 1$, and the differential of the spectral sequence $d^{1}: E_{n 1}^{1} \rightarrow E_{n-11}^{1}$ is exactly the Chevalley-Eilenberg differential $\partial: V \otimes \mathfrak{g}^{\wedge n-1} \rightarrow V \otimes \mathfrak{g}^{\wedge n-2}$. This implies an isomorphism

$$
E_{n 1}^{2}=H_{n-1}(\mathfrak{g}, V), \quad n \geqslant 0 .
$$

Thus, (4) and (5) imply the first part of the proposition.

Now, the direct calculus gives the following:

$$
E_{1 q}^{1}=H_{q}\left(E_{*}(V, 0,0)\right)=0 \quad \text { when } q \geqslant 2 .
$$

Therefore, we have an isomorphism

$$
H_{3}\left(\operatorname{Tot}\left(X_{* *}\right)\right)=E_{21}^{2}=H_{1}(\mathfrak{g}, V)
$$

and an epimorphism

$$
H_{4}\left(\operatorname{Tot}\left(X_{* *}\right)\right) \rightarrow E_{31}^{2}=H_{2}(\mathfrak{g}, V) .
$$

Question. What is the term $E_{22}^{2}$ ?

It would be interesting to give an answer to this question, since we have the following exact sequence:

$$
H_{5}(\Theta(V, \mathfrak{g})) \rightarrow H_{3}(\mathfrak{g}, V) \rightarrow E_{22}^{2} \rightarrow H_{4}(\Theta(V, \mathfrak{g})) \rightarrow H_{2}(\mathfrak{g}, V) \rightarrow 0 .
$$

Remark 4.2. A similar result for crossed modules of groups is proved in [10] using topological methods.

Corollary 4.3. For any Lie algebra $\mathfrak{g}$ and $\mathfrak{g}$-module $V$, there is the following eight term exact sequence 


$$
\begin{aligned}
H_{4}(V, \mathfrak{g}, 0) \longrightarrow H_{4}(\Theta(V, \mathfrak{g})) \longrightarrow H_{3}(\mathfrak{g}) \longrightarrow H_{3}(V, \mathfrak{g}, 0) \longrightarrow H_{1}(\mathfrak{g}, V) \longrightarrow \\
\longleftrightarrow H_{2}(\mathfrak{g}) \longrightarrow H_{2}(V, \mathfrak{g}, 0) \longrightarrow V /[V, \mathfrak{g}] \longrightarrow 0
\end{aligned}
$$

Proof. By the definition of $\Theta(V, \mathfrak{g})$ there is a long exact sequence of homology groups

$$
\cdots \rightarrow H_{n}(0, \mathfrak{g}, 0) \rightarrow H_{n}(V, \mathfrak{g}, 0) \rightarrow H_{n}(\Theta(V, \mathfrak{g})) \rightarrow H_{n-1}(0, \mathfrak{g}, 0) \rightarrow \cdots .
$$

For all $n \geqslant 0$, one has a natural isomorphism $H_{n}(0, \mathfrak{g}, 0)=H_{n}(\mathfrak{g})$ (see [4]). Moreover, by Proposition 3.1 we have

$$
H_{1}(0, \mathfrak{g}, 0)=H_{1}(V, \mathfrak{g}, 0)=\mathfrak{g} /[\mathfrak{g}, \mathfrak{g}] .
$$

Therefore, from the aforementioned long exact sequence we get the following exact sequence with eight terms:

$$
\begin{aligned}
H_{4}(V, \mathfrak{g}, 0) \longrightarrow H_{4}(\Theta(V, \mathfrak{g})) \longrightarrow H_{3}(\mathfrak{g}) \longrightarrow H_{3}(V, \mathfrak{g}, 0) \longrightarrow H_{3}(\Theta(V, \mathfrak{g})) \longrightarrow \\
\longleftrightarrow H_{2}(\mathfrak{g}) \longrightarrow H_{2}(V, \mathfrak{g}, 0) \longrightarrow H_{2}(\Theta(V, \mathfrak{g})) \longrightarrow 0 .
\end{aligned}
$$

Replacing $H_{3}(\Theta(V, \mathfrak{g}))$ and $H_{2}(\Theta(V, \mathfrak{g}))$ by $H_{1}(\mathfrak{g}, V)$ and $V /[V, \mathfrak{g}]$, respectively, we get the desired result.

Corollary 4.4. Let $V$ be a $\mathbf{k}$-module with trivial Lie bracket. Then

$$
H_{2}(V, 0,0)=V \quad \text { and } \quad H_{3}(V, 0,0)=0 .
$$

Proposition 4.5. Let $\mathbf{k}$ be a field and $1 / 2 \in \mathbf{k}$. Then

$$
H_{2}(V, \mathfrak{g}, 0)=H_{2}(\mathfrak{g}) \oplus V /[V, \mathfrak{g}] \quad \text { and } \quad H_{3}(V, \mathfrak{g}, 0)=H_{3}(\mathfrak{g}) \oplus H_{1}(\mathfrak{g}, V) .
$$

Proof. By Corollary 4.3 we have an exact sequence

$$
H_{2}(\mathfrak{g}) \rightarrow H_{2}(V, \mathfrak{g}, 0) \rightarrow V /[V, \mathfrak{g}] \rightarrow 0 .
$$

Denote by $\alpha$ the first homomorphism in the sequence (6). By Proposition 3.1 we have an epimorphism $\tau: H_{2}(V, \mathfrak{g}, 0) \rightarrow H_{2}(\mathfrak{g})$. It is easy to check that $\tau \alpha=1_{H_{2}(\mathfrak{g})}$. Hence, the sequence (6) splits

$$
H_{2}(V, \mathfrak{g}, 0)=H_{2}(\mathfrak{g}) \oplus V /[V, \mathfrak{g}] .
$$

Now, Corollary 4.3 and (7) imply the following exact sequence:

$$
H_{3}(\mathfrak{g}) \rightarrow H_{3}(V, \mathfrak{g}, 0) \rightarrow H_{1}(\mathfrak{g}, V) \rightarrow 0 .
$$

Denote by $\beta$ the natural homomorphism $H_{3}(\mathfrak{g}) \rightarrow H_{3}(V, \mathfrak{g}, 0)$. By Proposition 3.1 and (7) we have an epimorphism $\eta: H_{3}(V, \mathfrak{g}, 0) \rightarrow H_{3}(\mathfrak{g})$. Moreover, $\eta \beta=1_{H_{3}(\mathfrak{g})}$. This completes the proof.

Corollary 4.6. If $1 / 2 \in \mathbf{k}$, then there exists an epimorphism $H_{4}(V, \mathfrak{g}, 0) \rightarrow H_{2}(\mathfrak{g}, V)$.

Proof. By Corollary 4.3 and Proposition 4.5 we have a natural epimorphism $H_{4}(V, \mathfrak{g}, 0) \rightarrow H_{4}(\Theta(V, \mathfrak{g}))$. Moreover, by Proposition 4.1 there exists an epimorphism $H_{4}(\Theta(V, \mathfrak{g})) \rightarrow H_{2}(V, \mathfrak{g})$. 


\section{References}

[1] J.C. Baez and A.S. Crans, Higher-dimensional algebra. VI. Lie 2-algebras, Theor. Appl. Categ. 12 (2004), 492-538.

[2] J.C. Baez and A.D. Lauda, Higher-dimensional algebra. V. 2-groups, Theor. Appl. Categ. 12 (2004), 423-491.

[3] P. Carrasco, A.M. Cegarra and A.R. Grandjeán, (Co)homology of crossed modules, J. Pure Appl. Algebra 168 (2002), no. 2-3, 147-176.

[4] J.M. Casas, N. Inassaridze and M. Ladra, Homological aspects of Lie algebra crossed modules, Manuscripta Math. 131 (2010), no. 3-4, 385-401.

[5] J.M. Casas and M. Ladra, Homology of crossed modules in Lie algebras, Bull. Soc. Math. Belg. Sér. A 45 (1993), no. 1-2, 59-84.

[6] G. Donadze, N. Inassaridze, E. Khmaladze and M. Ladra, Cyclic homologies of crossed modules of algebras, J. Noncommut. Geom. 6 (2012), no. 4, 749771.

[7] G. Donadze and M. Ladra, Cotriple homology of crossed 2-cubes of Lie algebras, J. Algebra Appl. 12 (2013), no. 06, 1350007, 21 pp.

[8] G.J. Ellis, Crossed modules and their higher dimensional analogue, University of Wales, Ph.D. Thesis, 1984.

[9] G.J. Ellis, Homotopical aspects of Lie algebras, J. Austral. Math. Soc. Ser. A 54 (1993), no. 3, 393-419.

[10] A.R. Grandjeán, M. Ladra and T. Pirashvili, CCG-homology of crossed modules via classifying spaces, J. Algebra 229 (2000), no. 2, 660-665.

[11] C. Kassel and J.-L. Loday, Extensions centrales d'algèbres de Lie, Ann. Inst. Fourier (Grenoble) 32 (1982), no. 4, 119-142.

[12] F. Wagemann, On Lie algebra crossed modules, Comm. Algebra 34 (2006), no. $5,1699-1722$.

Guram Donadze gdonad@gmail.com

Kerala School of Mathematics, Kunnamangalam P.O., Kozhikode - 673 571, Kerala, India

Manuel Ladra manuel.ladra@usc.es

Department of Algebra, University of Santiago de Compostela, 15782 Santiago de Compostela, Spain 